\title{
Электрофизические свойства и механизмы переноса в тонких пленках материалов фазовой памяти на основе халькогенидных полупроводников квазибинарного разреза $\mathrm{GeTe}-\mathrm{Sb}_{2} \mathrm{Te}_{3}$
}

\author{
(ㄷ А.А. Шерченков ${ }^{1}$, С.А. Козюхин ${ }^{2,9}$, П.И. Лазаренко ${ }^{1}$, А.В. Бабич ${ }^{1}$, \\ Н.А. Богословский ${ }^{3}$, И.В. Сагунова ${ }^{1}$, Е.Н. Редичев ${ }^{1}$ \\ ${ }^{1}$ Национальный исследовательский университет „Московский институт электронной техники“, \\ 124498 Москва, Зеленоград, Россия \\ ${ }^{2}$ Институт общей и неорганической химии им. Н.С. Курнакова Российской академии наук, \\ 119991 Москва, Россия \\ ${ }^{3}$ Физико-технический институт им. А.Ф. Иофффе Российской академии наук, \\ 194021 Санкт-Петербург, Россия \\ ๑ E-mail: sergkoz@igic.ras.ru
}

(Получена 12 апреля 2016 г. Принята к печати 18 апреля 2016 г.)

\begin{abstract}
Исследованы температурные зависимости удельного сопротивления и вольт-амперных характеристик тонких пленок материалов фазовой памяти на основе халькогенидных полупроводников квазибинарного разреза $\mathrm{GeTe}-\mathrm{Sb}_{2} \mathrm{Te}_{3}: \mathrm{Ge}_{2} \mathrm{Sb}_{2} \mathrm{Te}_{5}, \mathrm{GeSb}_{2} \mathrm{Te}_{4}$ и $\mathrm{GeSb}_{4} \mathrm{Te}_{7}$. Изучено влияние изменения состава по линии квазибинарного разреза на электрофизические характеристики и механизмы переноса тонких пленок. Установлено наличие трех диапазонов с различной зависимостью между током и напряжением. Оценено положение и концентрация энергетических уровней, контролирующих перенос носителей. Полученные результаты показывают, что электрофизические свойства тонких пленок могут существенно изменяться при движении вдоль линии квазибинарного разреза $\mathrm{GeTe}-\mathrm{Sb}_{2} \mathrm{Te}_{3}$, что важно для целенаправленной оптимизации технологии фазовой памяти.
\end{abstract}

DOI: 10.21883/FTP.2017.02.44096.8270

\section{1. Введение}

В последнее время активно совершенствуются старые и разрабатываются новые виды энергонезависимой памяти. Одним из наиболее перспективных видов энергонезависимых запоминающих устройств нового поколения считается фазовая память [1,2], которая по ряду показателей либо уже превосходит, либо в перспективе может превзойти существующие и разрабатываемые виды памяти. Более того, она претендует на то, чтобы стать универсальной, сочетающей в себе достоинства энергонезависимой и быстродействующей динамической памятей, обладающей к тому же повышенной радиационной стойкостью.

Принцип действия фазовой памяти основан на обратимом фазовом переходе материала из аморфного состояния в кристаллическое и обратно под действием внешних низкоэнергетических воздействий (лазерного излучения или электрического тока). Переход сопровождается резким изменением оптических и/или электрических характеристик, что дает возможность разделить логические „, $0^{\star \varsigma}$ и „1“. В электрической фазовой памяти кристаллическое состояние с низким сопротивлением ( кОм) соответствует логической „1“, а аморфное состояние с высоким сопротивлением ( МОм $)$ логическому „О“.

За последние несколько лет в технологии фазовой памяти достигнут заметный прогресс. Однако, несмотря на успехи, электрическая фазовая память не стала широко распространенной. Объясняется это неотработанностью технологии и наличием ряда нерешенных научно-технических проблем. К тому же совершенствование технологии усложняется наличием специфических требований к свойствам материалов фазовой памяти, которые зачастую противоречат друг другу и поэтому могут быть лишь компромиссными. В связи с этим, лишь ограниченный круг материалов пригоден для устройств фазовой памяти, к которым относятся халькогенидные полупроводники.

Современный этап развития технологии электрической фазовой памяти связан с использованием тонкопленочного халькогенидного соединения $\mathrm{Ge}_{2} \mathrm{Sb}_{2} \mathrm{Te}_{5}$. $\mathrm{B}$ настоящий момент на основе $\mathrm{Ge}_{2} \mathrm{Sb}_{2} \mathrm{Te}_{5}$ получены наилучшие параметры устройств фазовой памяти: время переключения 50-100 нс, число циклов перезаписи по разным данным от $10^{5}$ до $10^{13}$. Однако при том, что $\mathrm{Ge}_{2} \mathrm{Sb}_{2} \mathrm{Te}_{5}$ наиболее широко используется на практике, его свойства не являются оптимальными как по скорости и температуре кристаллизации, так и по электрофизическим характеристикам.

Для дальнейшего совершенствования технологии фазовой памяти и получения ячеек с улучшенными характеристиками необходимо иметь возможность целенаправленно изменять свойства материала. Одним из распространенных способов управления свойствами полупроводниковых материалов является легирование. Однако для халькогенидных стеклообразных полупроводников легирование не является эффективным методом, 
что обусловлено большой плотностью состояний в щели подвижности, эффективно закрепляющих уровень Ферми в середине щели подвижности [3].

В то же время $\mathrm{Ge}_{2} \mathrm{Sb}_{2} \mathrm{Te}_{5}$ находится на линии квазибинарного разреза $\mathrm{Sb}_{2} \mathrm{Te}_{3}-\mathrm{GeTe}$, на котором имеются также еще два соединения $\mathrm{GeSb}_{2} \mathrm{Te}_{4}$ и $\mathrm{GeSb}_{4} \mathrm{Te}_{7}$. Все три соединения $\mathrm{Ge}_{2} \mathrm{Sb}_{2} \mathrm{Te}_{5}$ (GST25), $\mathrm{GeSb}_{2} \mathrm{Te}_{4}$ (GST124) и $\mathrm{GeSb}_{4} \mathrm{Te}_{7}$ (GST147) отличаются соотношениями $(\mathrm{GeTe})_{n}\left(\mathrm{Sb}_{2} \mathrm{Te}_{3}\right)_{m}$, где $m: n=2: 1 ; 1: 1$; $1: 2$ соответственно. Они образуют гомологический ряд с закономерным изменением свойств. В работе [4] показано, что при движении по линии квазибинарного разреза $\mathrm{GeTe}-\mathrm{Sb}_{2} \mathrm{Te}_{3}$ в сторону $\mathrm{Sb}_{2} \mathrm{Te}_{3}$ (т.е. от $\mathrm{Ge}_{2} \mathrm{Sb}_{2} \mathrm{Te}_{5}$ к $\mathrm{GeSb}_{2} \mathrm{Te}_{4}$ и далее к $\mathrm{GeSb}_{4} \mathrm{Te}_{7}$ ) происходит заметное уменьшение температуры, энергии активации и времени фазового перехода из аморфного состояния в кристаллическое. В то же время, влияние изменения состава по квазибинарному разрезу $\mathrm{GeTe}-\mathrm{Sb}_{2} \mathrm{Te}_{3}$ на электрофизические характеристики и механизмы переноса тонких пленок остаются не до конца изученными. Понимание данных вопросов имеет важное практическое значение, поскольку является необходимым условием для разработки эффективных методов совершенствования и целенаправленной оптимизации технологии фазовой памяти, в частности, за счет управления электрофизическими свойствами халькогенидных программируемых материалов.

В связи с этим цель данной работы заключалась в выяснении влияния состава материалов фазовой памяти, находящихся на линии квазибинарного разреза $\mathrm{GeTe}-\mathrm{Sb}_{2} \mathrm{Te}_{3}$, на электрофизические характеристики и механизмы переноса тонких пленок.

\section{2. Методика эксперимента}

Синтез материалов $\mathrm{Ge}_{2} \mathrm{Sb}_{2} \mathrm{Te}_{5}, \mathrm{GeSb}_{2} \mathrm{Te}_{4}$ и $\mathrm{GeSb}_{4} \mathrm{Te}_{7}$ проводился из материалов полупроводниковой степени чистоты [5]. Для нанесения тонких пленок использовалось вакуумно-термическое испарение синтезированного материала. Температура подложки во время осаждения не превышала $50^{\circ} \mathrm{C}$.

Согласно данным рентгенофазового анализа (Rigaku D/MAX, $\mathrm{CuK}_{\alpha} \lambda=15481$ нм), синтезированный материал являлся поликристаллическим и имел тригональную модификацию. Условия осаждения обеспечили получение тонких пленок в аморфном состоянии, отжиг которых при температурах выше $150^{\circ} \mathrm{C}$ приводил к кристаллизации слоев.

Состав тонких пленок исследовался методом резерфордовского обратного рассеяния $\left(E_{d}=1.0, E_{a}=2.7 \mathrm{MэB}\right.$, $\left.\varphi=135^{\circ}\right)$ и показал, что отклонение состава тонких пленок от заданного не превышало точности метода измерения $( \pm 5 \%)$.

Для проведения исследований электрофизических характеристик тонких пленок были изготовлены планарные образцы, которые представляли собой окислен- ную кремниевую подложку со сформированными на ее поверхности алюминиевыми электродами. Поверх межэлектродных промежутков (7 мкм) осаждались халькогенидные тонкие пленки, покрываемые в дальнейшем защитным слоем $\mathrm{SiO}_{x}$. Контроль геометрических параметров электродов проводился на оптическом микроскопе (Carl Zeiss Axiovert $40 \mathrm{MAC})$, растровом электронном микроскопе (Carl Zeiss NVision 40) и атомно-силовом микроскопе (ACM NT-MDT SolverPro). Толщины исследуемых пленок находились в диапазоне от 60 до 80 нм.

Электрофизические характеристики тонких пленок исследовались на специализированном стенде, представляющем собой двухэлектродную схему на основе блока управления напряжением NI6008 и пикоамперметра KEITHLEY 6486. Измерение температурных зависимостей удельного сопротивления тонких пленок проводилось при напряжении $0.7 \mathrm{~B}\left(E=1 \cdot 10^{3} \mathrm{~B} / \mathrm{cm}\right)$ в диапазоне от комнатной температуры до $200^{\circ} \mathrm{C}$ при скорости нагрева $1^{\circ} \mathrm{C} /$ мин. Вольт-амперные характеристики (BAX) исследовались в диапазоне $0.1-10 \mathrm{~B}$ с шагом $0.1 \mathrm{~B}$ и от комнатной температуры до $70^{\circ} \mathrm{C}$ c шагом $10^{\circ} \mathrm{C}$.

\section{3. Экспериментальные результаты и обсуждение}

На рис. 1 представлены ВАХ тонкой пленки GST225 при разных температурах нагрева. Аналогичные BAX получены также для тонких пленок $\mathrm{GeSb}_{2} \mathrm{Te}_{4}$ и $\mathrm{GeSb}_{4} \mathrm{Te}_{7}$.

На всех исследованных ВАХ можно выделить три характерных участка с различными зависимостями тока от напряжения: при слабых $\left(E<1 \cdot 10^{3} \mathrm{~B} / \mathrm{cm}\right)$, средних $\left(1 \cdot 10^{3}<E<1 \cdot 10^{4} \mathrm{~B} / \mathrm{cm}\right)$ и сильных $\left(E>1 \cdot 10^{4} \mathrm{~B} / \mathrm{cm}\right)$ напряженностях электрических полей. Наличие различных зависимостей тока от напряжения связано с доминированием разных механизмов переноса носителей заряда [6-8].

\section{1. Область слабых напряженностей электрических полей}

В области слабых полей зависимости тока от напряжения близки к линейной, что является типичным для халькогенидных стеклообразных полупроводников [9].

На рис. 2 представлены температурные зависимости удельного сопротивления тонких пленок GST225, GST124 и GST147.

Видно, что на зависимостях можно выделить три температурных диапазона. От комнатной температуры до $\sim 100^{\circ} \mathrm{C}$ наблюдаются экспоненциальные температурные зависимости, что указывает на активационный характер проводимости тонких пленок в аморфном состоянии. При этом зависимости описываются следую- 
Таблица 1. Характеристики тонких пленок материалов квазибинарного разреза $\mathrm{Sb}_{2} \mathrm{Te}_{3}-\mathrm{GeTe}$

\begin{tabular}{|c|c|c|c|c|c|}
\hline \multirow{2}{*}{ Состав } & $T_{x}-T_{y},{ }^{\circ} \mathrm{C}$ & $\Delta T_{n},{ }^{\circ} \mathrm{C}$ & \multirow{2}{*}{$\rho_{a}, \mathrm{OM} \cdot \mathrm{cm}$} & \multirow{2}{*}{$\rho_{\mathrm{cr}}, \mathrm{OM} \cdot \mathrm{cm}$} & \multirow{2}{*}{$\rho_{a} / \rho_{\mathrm{cr}}$} \\
\hline & \multicolumn{2}{|c|}{$\rho \sim T$} & & & \\
\hline $\begin{array}{l}\text { GST147 } \\
\text { GST124 } \\
\text { GST225 }\end{array}$ & $\begin{array}{l}100-118 \\
122-128 \\
132-139\end{array}$ & $\begin{array}{r}18 \\
6 \\
7\end{array}$ & $\begin{array}{r}4.9 \cdot 10^{4} \\
1 \cdot 10^{5} \\
2.8 \cdot 10^{4}\end{array}$ & $\begin{array}{l}2.6 \\
3.8 \\
1.0\end{array}$ & $\begin{array}{l}1.9 \cdot 10^{4} \\
2.6 \cdot 10^{4} \\
2.8 \cdot 10^{4}\end{array}$ \\
\hline
\end{tabular}

щим выражением:

$$
1 / \rho=\sigma=\sigma_{0} \exp \left(-\frac{E_{\mathrm{F}}}{k T}\right),
$$

где $\sigma_{0}-$ предэкспоненциальный множитель, $E_{a}-$ энергия активации проводимости, $k-$ постоянная Больцмана.

В диапазоне температур от $\sim 100$ до $\sim 140^{\circ} \mathrm{C}$ происходит резкое падение удельного сопротивления тонких пленок на несколько порядков. Результаты рентгенофазового анализа указывают на то, что такое изменение

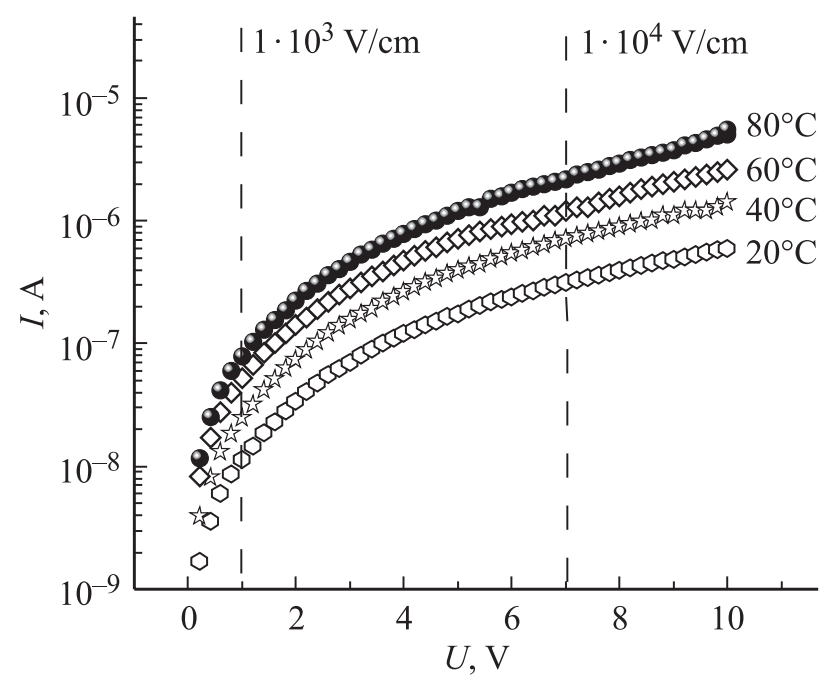

Рис. 1. Вольт-амперные характеристики аморфной тонкой пленки GST225 в диапазоне температур от 20 до $80^{\circ} \mathrm{C}$.

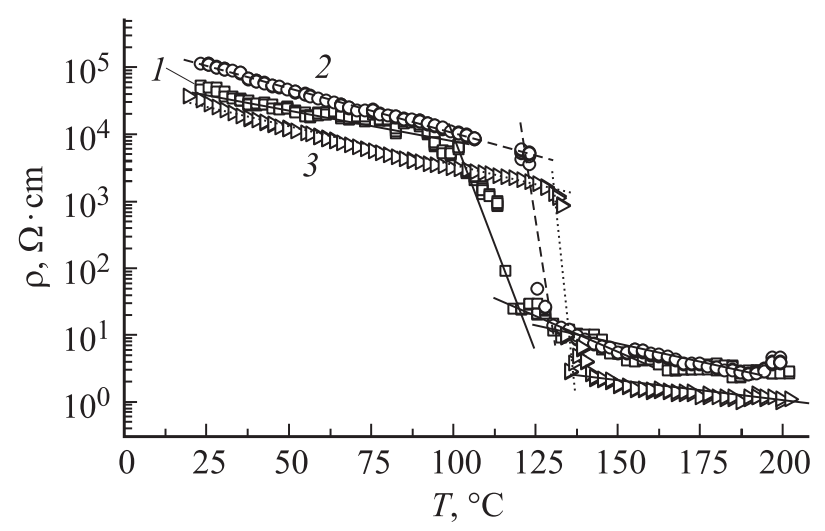

Рис. 2. Температурные зависимости удельного сопротивления тонких пленок: 1 - GST147, 2 - GST124, 3 - GST225.
Таблица 2. Энергетические параметры тонких пленок материалов квазибинарного разреза $\mathrm{Sb}_{2} \mathrm{Te}_{3}-\mathrm{GeTe}$

\begin{tabular}{l|c|c|c|c|c}
\hline Материал & $E_{g}$, эВ & $E_{g} / 2$, эВ & $E_{a}$, эВ & $E_{0}$, эВ & $\sigma_{0}, \mathrm{OM}^{-1} \cdot \mathrm{cm}^{-1}$ \\
\hline $\mathrm{GeSb}_{4} \mathrm{Te}_{7}$ & 0.50 & 0.25 & 0.22 & 0.09 & 0.1 \\
$\mathrm{GeSb}_{2} \mathrm{Te}_{4}$ & 0.54 & 0.27 & 0.28 & 0.08 & 1.0 \\
$\mathrm{Ge}_{2} \mathrm{Sb}_{2} \mathrm{Te}_{5}$ & 0.61 & 0.30 & 0.29 & 0.13 & 2.2
\end{tabular}

удельного сопротивления связано с кристаллизацией тонких пленок. Полученные результаты согласуются с литературными данными $[2,4]$, согласно которым для соединений на квазибинарном разрезе $\mathrm{Sb}_{2} \mathrm{Te}_{3}-\mathrm{GeTe}$ фазовые переходы из аморфной фазы в кубическую структуру типа $\mathrm{NaCl}(f c c)$, которая является низкотемпературной кристаллической метастабильной модификацией, происходят в близком температурном диапазоне и сопровождаются резким падением удельного сопротивления.

При более высоких температурах для исследованных тонких пленок наблюдаются слабые экспоненциальные зависимости удельного сопротивления от температуры, что характерно для халькогенидных слоев в кристаллическом состоянии [10].

Результаты анализа температурных зависимостей удельного сопротивления представлены в табл. 1 , где $T_{x}$ и $T_{y}$ - температуры начала и окончания фазового перехода, $\Delta T_{n}=T_{x}-T_{y}, \rho_{a}$ и $\rho_{\mathrm{cr}}-$ удельные сопротивления при 20 и $250^{\circ} \mathrm{C}$ соответственно.

Из представленных данных в табл. 1 и на рис. 2 видно, что при изменении соотношения $(\mathrm{GeTe})_{n}\left(\mathrm{Sb}_{2} \mathrm{Te}_{3}\right)_{m}$ от $n: m=1: 2$ (GST147) к $1: 1$ (GST124) и далее к $2: 1$ (GST225), т.е. с ростом относительного содержания $\mathrm{GeTe}$ происходит увеличение температуры начала фазового перехода, что согласуется с данными [4], а также уменьшение температурного диапазона фазового перехода $\Delta T_{n}$. Уменьшение температуры кристаллизации при движении от GST225 к GST147 обусловлено увеличением доли более слабых химических связей $\mathrm{Sb}-\mathrm{Te}$ (277.5 кДж/моль [11]) по сравнению с более сильными химическими связями $\mathrm{Ge}-\mathrm{Te}$ (402 кДж/моль [11]), что совпадает с данными [4].

Аппроксимация зависимостей, представленных на рис. 2, в первом диапазоне с использованием выражения (1) позволила оценить энергию активации проводимости. Энергетические параметры тонких пленок исследуемых составов представлены в табл. 2, где $E_{g}-$ 


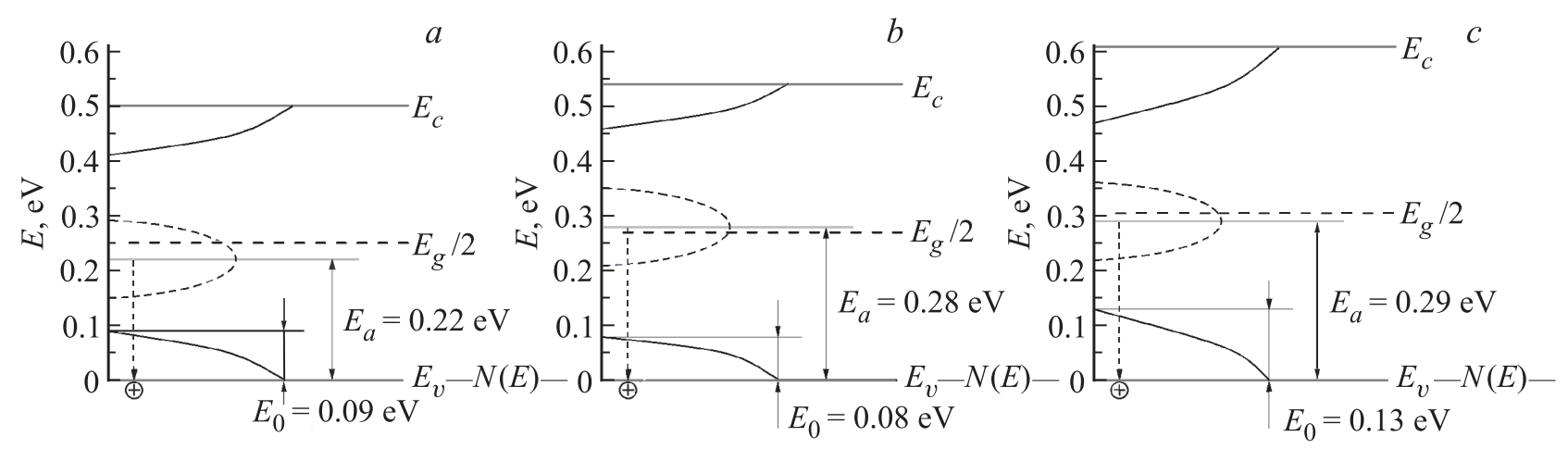

Рис. 3. Энергетические диаграммы тонких пленок: $a$ - GST147, $b-\mathrm{GST} 124, c-\mathrm{GST} 225$. Штриховой линией схематически отображено наличие зоны глубоких состояний в середине щели по подвижности.

ширина оптической щели, $E_{0}$ - энергия Урбаха. Величины ширины оптической щели и энергии Урбаха составов взяты из работы [12], погрешность составляет \pm 0.01 эВ. Полученные результаты согласуются с литературными данными [13].

В работе[12] показано, что на спектральных зависимостях коэффициентов поглощения для исследуемых нами аморфных тонких пленок GST225, GST124 и GST147 наблюдаются участки с экспоненциальными зависимостями. Данный результат с учетом активационного характера проводимости тонких пленок позволяет сделать вывод, что для исследуемых слоев халькогенидных стеклообразных полупроводников применима модель строения энергетических зон Мотта-Дэвиса (МД) [14]. Характерными чертами этой модели являются узкие хвосты локализованных состояний на краях валентной зоны и зоны проводимости, распространяющиеся в щель по подвижности и имеющие экспоненциальные распределения плотности состояний, а также граничные энергии, отделяющие локализованные состояния от распространенных состояний в зонах. Уровень Ферми в модели МД закрепляется в зоне локализованных состояний вблизи середины щели по подвижности, обязанных своим происхождением дефектам. В этом случае экспоненциальные участки на спектральных зависимостях коэффициентов поглощения обусловлены оптическими переходами с участием экспоненциально распределенных состояний хвостов зон.

Наибольшей протяженностью хвостов в глубь щели по подвижности обладают тонкие пленки GST225 $\left(E_{0}=0.13\right.$ эВ). При этом значения $E_{0}$ для тонких пленок GST124 (0.08 эB) и GST147 (0.09 эВ) близки. Полученные значения параметра Урбаха указывают на то, что тонкие пленки GST124 и GST147 обладают меньшей степенью разупорядоченности структуры по сравнению c GST225.

Основываясь на модели МД и с учетом экспериментальных результатов, были построены зонные энергетические диаграммы для аморфных тонких пленок GST (рис. 3). При этом учитывалось, что измерения термо-
ЭДС, проведенные на исследуемых тонких пленках, показали, что GST имеют дырочный тип проводимости [15].

Анализ диаграмм выявил, что полученные значения энергии активации проводимости для GST близки к середине щели по подвижности $\left(E_{g} / 2\right)$, кроме состава GST124, для которого вычисленная энергия активации оказалась несколько выше $E_{g} / 2$.

Наблюдаемое увеличение энергии активации проводимости при переходе от GST147 к GST225 связано с изменением ширины оптической щели и плотности состояний в ней. Уменьшение ширины оптической щели обусловлено уменьшением энергии дна зоны проводимости вследствие уширения зоны антисвязывающих состояний и роста ее заселенности при увеличении концентрации структурных единиц $\mathrm{SbTe}_{3 / 2}$ с более слабыми химическими связями [12].

Близость энергий активации проводимости для всех составов к середине щели по подвижности позволяет предположить, что существенный вклад в проводимость вносит перенос носителей заряда по делокализованным состояниям валентной зоны. Однако в работе [16] отмечается, что для большинства неупорядоченных полупроводников при переносе носителей заряда по делокализованным состояниям предэкспоненциальный множитель $\sigma_{0}$ должен лежать в диапазоне от 10 до $10^{3} \mathrm{OM}^{-1} \cdot \mathrm{cm}^{-1}$. Полученные нами значения $\sigma_{0}$ для материалов GST существенно меньше данных значений. В связи с этим для описания полученных зависимостей $\sigma$ от $1 / k T$ нами использовалась двухканальную модель проводимости, предложенная Нагельсом [16]. Согласно модели, в проводимость ХСП р-типа могут вносить одновременный вклад носители, перемещающиеся по локализованным состояниям хвоста валентной зоны и распространенным состояниям валентной зоны:

$$
\begin{aligned}
\sigma= & \sigma_{0}^{\prime} \exp \left[-\left(E_{\mathrm{F}}-E_{\mathrm{V}}\right) / k T\right] \\
& +\sigma_{0 h} \exp \left[-\left(E_{\mathrm{F}}-E_{0}+W\right) / k T\right],
\end{aligned}
$$

где первое слагаемое соответствует переносу носителей заряда по распространенным состояниям, а второе прыжковому механизму переноса по локализованным 
Таблица 3. Энергетические параметры тонких пленок на основе материалов квазибинарного разреза $\mathrm{Sb}_{2} \mathrm{Te}_{3}-\mathrm{GeTe}$

\begin{tabular}{c|c|c|c|c|c|c|c}
\hline \multirow{2}{*}{ Материал } & \multicolumn{3}{|c|}{ Варьируемые величины } & \multicolumn{3}{c}{ Фиксированные величины } \\
\cline { 2 - 7 } & $\sigma_{0}^{\prime}, \mathrm{OM}^{-1} \cdot \mathrm{cm}^{-1}$ & $\sigma_{0 h}, \mathrm{OM}^{-1} \cdot \mathrm{cm}^{-1}$ & $W$, эB & $E_{g}$, эB & $E_{g} / 2$, эВ & $E_{f}$, эB & $E_{0}$, эB \\
\hline GST147 & $0.08-0.12$ & $0.0008-0.0012$ & $0.005-0.015$ & 0.50 & 0.25 & 0.22 & 0.09 \\
GST124 & $0.45-0.60$ & $0.0045-0.0060$ & $0.005-0.015$ & 0.54 & 0.27 & 0.28 & 0.08 \\
GST225 & $2.0-3.0$ & $0.01-0.02$ & $0.005-0.015$ & 0.61 & 0.30 & 0.29 & 0.13
\end{tabular}

состояниям хвоста зоны ( $W$ - энергия, необходимая для совершения прыжка).

Возможность применения двухканальной модели была продемонстрирована Ван-дер-Пласом, Бубе и Нагельсом для описания материалов фазовой памяти предыдущего поколения систем $\mathrm{Ge}_{x} \mathrm{Te}_{1-x}$ и $\mathrm{Si}-\mathrm{Te}-\mathrm{As}-\mathrm{Ge}$ (STAG) $[17,18]$.

При аппроксимации экспериментальных зависимостей с использованием уравнения (2) изменялись параметры $\sigma_{0}, \sigma_{0 h}$ и $W$, в качестве $E_{f}$ использовалось полученное значение энергии активации проводимости, а в качестве $E_{g}$ и $E_{0}-$ результаты исследования оптических спектров пропускания тонких пленок (см. табл. 2).

На рис. 4 представлены температурные зависимости проводимости, из которых видно, что результаты аппроксимации и экспериментальные данные хорошо согласуются.

Следует, однако, отметить, что использование уравнения (2) для аппроксимации температурной зависимости проводимости не позволяет однозначно определить параметры $\sigma_{0}, \sigma_{0 h}$ и $W$, а лишь дает возможность оценить диапазоны, в которых они находятся. Результаты представлены в табл. 3 .

Нагельс с соавт. [16] приводят следующие значения для предэкспоненциальных множителей: $\sigma_{0}^{\prime}=$ $=3000 \mathrm{OM}^{-1} \cdot \mathrm{cm}^{-1}$ и $\sigma_{0 h}=90 \mathrm{OM}^{-1} \cdot \mathrm{cm}^{-1}$. Наши ре-

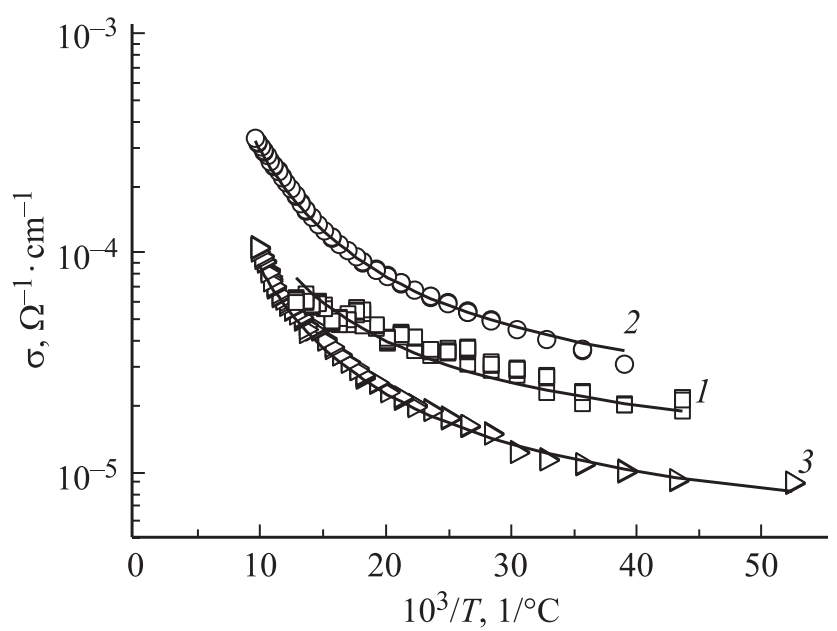

Рис. 4. Зависимости проводимости от 1000/T: 1 - GST147, 2 - GST124, 3 - GST225. Маркерами отмечены экспериментальные точки, сплошными линиями - результаты аппроксимации. зультаты аппроксимации показали, что аналогичные значения, полученные с использованием двухканальной модели (см. табл. 3), отличаются от значений в работе [16]. В то же время разница между оцененными значениями $\sigma_{0}^{\prime}$ и $\sigma_{0 h}$ составляет несколько порядков, что коррелирует с данными [16] и согласуется с моделью МД, в которой происходит резкое падение подвижности носителей при энергии, разделяющей распространенные и локализованные состояния валентной зоны. Различие в значениях предэкспоненциальных факторов может быть объяснено особенностями физико-химических свойств материалов GST, например, тонкие пленки GST обладают чрезвычайно высокой скоростью, стабильностью и воспроизводимостью переключения из аморфного состояния в кристаллическое при воздействии импульсов электрического тока $[4,10,19]$.

\section{2. Область средних напряженностей электрических полей}

В области средних напряженностей электрического поля наблюдается степенная зависимость между током и напряжением

$$
j=K V^{m}
$$

где $K-$ коэффициент, зависящий от толщины пленки, плотности распределения ловушек и проводимости исследуемых материалов.

Степенная зависимость тока от напряжения характерна для токов, ограниченных пространственным зарядом (ТОПЗ) [20,21]. Согласно классической теории диэлектриков [21], при приложении электрического поля к диэлектрику возникает протекание тока за счет электронов, инжектированных из катода. Для ТОПЗ в идеальном диэлектрике характерна следующая зависимость плотности тока от напряжения [21]:

$$
j \approx \varepsilon_{0} \varepsilon \mu \frac{V^{2}}{L^{3}},
$$

где $\varepsilon_{0}$ - диэлектрическая постоянная, $\varepsilon-$ диэлектрическая проницаемость образца, $\mu-$ подвижность носителей заряда, $L-$ толщина пленки.

Квадратичная зависимость тока от напряжения соответствует идеальному ТОПЗ и наблюдается в полупроводниках как при переносе по зонным состояниям, так и при наличии захвата носителей заряда на дискретный уровень локализованных состояний [22]. 
Однако аппроксимация ВАХ исследованных нами тонких пленок показала, что для всех образцов при комнатной температуре наблюдаются степенные зависимости со степенями менее 2. Это связано с тем, что, как показано в теории Роуза [21,22], наличие ловушек в запрещенной зоне может оказывать существенное влияние на протекание инжекционных токов. Кроме того, Лампертом было показано [21], что в зависимости от положения ловушек и их распределения может наблюдаться отклонение показателя степени от двух. Отклонения степенной зависимости от квадратичной экспериментально наблюдалось в ряде работ [23-25], посвященных исследованию неупорядоченных полупроводников, в том числе и ХСП.

Кроме того, установлено, что для тонких пленок на основе соединений квазибинарного разреза $\mathrm{Sb}_{2} \mathrm{Te}_{3}-\mathrm{GeTe}$ наблюдаются экспоненциальные зависимости коэффициента $K$ от $1 / k T$.

$\mathrm{B}$ соответствии с работами $[21,26]$ нелинейность ВАХ в полупроводниках $p$-типа, связанная с наличием захвата инжектированных носителей заряда на дискретный уровень локализованных состояний, может быть описана выражением

$$
j \approx \theta \varepsilon_{0} \varepsilon \mu \frac{V^{2}}{L^{3}},
$$

где $\theta-$ коэффициент, характеризующий соотношение концентраций свободных носителей и носителей, захваченных на ловушках.

Можно показать, что

$$
\theta=\frac{p}{p_{t}} \approx \frac{N_{V} g_{i}}{N_{t}} \exp \left(\frac{E_{V}-E_{t}}{k T}\right),
$$

где $p$ и $p_{t}$ - концентрации свободных носителей и носителей, захваченных на ловушках, соответственно, $N_{V}$ - эффективная плотность состояний в валентной зоне; $E_{V}$ - потолок валентной зоны, $N_{t}$ и $E_{t}-$ концентрация и энергия уровня ловушек, $g_{i}-$ степень вырождения уровня ловушек, принимаемая равной $1 / 2$.

В результате для коэффициента $K$ можно записать:

$$
K=K_{0} \exp \left(-\frac{E_{t}-E_{V}}{k T}\right),
$$

где

$$
K_{0}=\varepsilon_{0} \varepsilon \mu \frac{N_{V} g_{t}}{N_{t} L^{3}}
$$

Предполагая, что подвижность имеет слабый активационный характер, используя выражение (7) и определив тангенс угла наклона зависимости $K$ от $1 / k T$, было оценено положение уровня $\left(E_{t}\right)$, наиболее сильно ограничивающего протекание тока, и значение предэкспоненциального фактора $K_{0}$.

Далее, используя уравнение (7) и полагая, что $\varepsilon \approx 16$ [27], $\mu \approx 20 \mathrm{~cm}^{2} / \mathrm{B} \cdot \mathrm{c}[28], N_{v} \approx 10^{19} \mathrm{~cm}^{-3}[26]$ и $g_{i}=1 / 2$, были оценены плотности состояний $N_{t}$ для уровней $E_{t}$ исследованных тонких пленок (табл. 4).
Таблица 4. Энергетические параметры тонких пленок на основе материалов квазибинарного разреза $\mathrm{Sb}_{2} \mathrm{Te}_{3}-\mathrm{GeTe}$

\begin{tabular}{c|c|c|c}
\hline Соединение & $E_{a}$, эB & $E_{t},{ } \mathrm{~B}$ & $N_{t}, \mathrm{~cm}^{-3}$ \\
\hline $\mathrm{GeSb}_{4} \mathrm{Te}_{7}$ & 0.22 & 0.20 & $2 \cdot 10^{16}$ \\
$\mathrm{GeSb}_{2} \mathrm{Te}_{4}$ & 0.28 & 0.27 & $9 \cdot 10^{14}$ \\
$\mathrm{Ge}_{2} \mathrm{Sb}_{2} \mathrm{Te}_{5}$ & 0.29 & 0.28 & $8 \cdot 10^{14}$
\end{tabular}

Из данных табл. 4 видно, что захват основных носителей заряда в области средних полей осуществляется уровнем $E_{t}$, расположенным несколько ближе к потолку валентной зоны по сравнению с активационным уровнем $E_{a}$, определенным для области слабых полей.

При низких напряжениях инжектируемые из алюминиевого контакта дырки захватываются глубокими ловушками, но за счет наличия равновесных носителей заряда зависимость тока от напряжения остается линейной до тех пор, пока средняя концентрация инжектированных неравновесных свободных носителей не становится сравнимой с концентрацией тех носителей, которые освобождены термически. С увеличением концентрации инжектированных свободных дырок в валентной зоне положение квазиуровня Ферми начинает смещаться ближе к валентной зоне. Таким образом, положение уровня, наиболее сильно ограничивающего протекание тока, несколько ближе к потолку валентной зоны, чем активационный уровень $E_{a}$ (см. табл. 4$)$.

При движении по линии квазибинарного разреза от $\mathrm{GeTe}$ к $\mathrm{Sb}_{2} \mathrm{Te}_{3}$ наблюдается изменение положения уровня $E_{t}$, что может быть связано с уменьшением ширины щели по подвижности и перераспределением плотности состояний в щели по подвижности. В частности, при переходе от GST225 к GST147 плотность состояний $N_{t}$ увеличивается с $8 \cdot 10^{14}$ до $2 \cdot 10^{16} \mathrm{~cm}^{-3}$, что коррелирует с изменением щели по подвижности в этом же направлении. Это объясняется увеличением концентрации более слабых связей $\mathrm{Sb}-\mathrm{Te}$ по сравнению с более сильными связями $\mathrm{Ge}-\mathrm{Te}$.

\section{3. Область высоких напряженностей электрических полей}

При $E>1 \cdot 10^{4} \mathrm{~B} / \mathrm{cm}$ для всех соединений появляется область более сильного возрастания тока по сравнению с участком средних напряженностей электрического поля. Это может быть обусловлено ионизацией мелких локализованных состояний [6], а также эффектом ПулаФренкеля, приводящим к уменьшению потенциального барьера при увеличении приложенного напряжения. Однако экспериментальные зависимости могут быть описаны как экспоненциальной, так и степенной зависимостью, с высокими коэффициентами детерминации. В связи с этим необходимо проведение дополнительных исследований. 


\section{4. Заключение}

Таким образом, исследовано влияние состава материалов фазовой памяти, находящихся на линии квазибинарного разреза $\mathrm{GeTe}-\mathrm{Sb}_{2} \mathrm{Te}_{3}$, на электрофизические характеристики и механизмы переноса тонких пленок. Установлено наличие трех диапазонов с различной зависимостью между током и напряжением. Оценены положение и концентрация энергетических уровней, контролирующих перенос носителей. Полученные результаты показывают, что электрофизические свойства тонких пленок могут существенно изменяться при движении вдоль линии квазибинарного разреза $\mathrm{GeTe}-\mathrm{Sb}_{2} \mathrm{Te}_{3}$, что важно для целенаправленной оптимизации технологии фазовой памяти.

Работа выполнена при поддержке Министерства образования и науки Российской Федерации (ФЦП, проект № 14.578.21.0085, уникальный идентификатор прикладных научных исследований (проекта) RFMEFI57814X0085).

\section{Список литературы}

[1] G.W. Burr, M.J. Breitwisch, M. Franceschini, D. Garetto, K. Gopalakrishnan, B. Jackson, B. Kurdi, C. Lam, L.A. Lastras, A. Padilla, B. Rajendran, S. Raoux, R.S. Shenoy. J. Vac. Sci. Technol. B, 28 (2), 223 (2010).

[2] S. Raoux, W. Wełnic, D. Ielmini. Chem. Rev., 110, 240 (2010).

[3] N.F. Mott, E.A. Davis. Electron Processes in Non-Crystalline Materials. (Oxford. Clarendon Press, 1979).

[4] N. Yamada, E. Ohno, K. Nishiuchi, N. Akahira, M. Takao. J. Appl. Phys., 69, 2849 (1991).

[5] С.А. Козюхин, А.А. Шерченков, Е.В. Горшкова и др. Неорг. матер., 45 (4), 408 (2009).

[6] M. Nardone, M. Simon, I.V. Karpov, V.G. Karpov. J. Appl. Phys., 112, 071101 (2012).

[7] P. Lazarenko, A. Sherchenkov, S. Kozyukhin, Y. Shtern, S.P. Timoshenkov, D.G. Gromov, E.N. Redichev. SPIE Proc., 9440, 944006 (2014).

[8] Н.А. Богословский, К.Д. Цэндин. ФТП, 43 (10), 1378 (2009).

[9] Э.А. Лебедев, С.А. Козюхин, Н.Н. Константинова, Л.П. Казакова. ФТП, 43 (10), 1383 (2009).

[10] H.-S.P. Wong, S. Raoux, S.-B. Kim, J. Liang, J.P. Reifenberg, B. Rajendran, M. Asheghi, K.E. Goodson. Proc. IEEE, 98 (12), 2201 (2010).

[11] J.A. Dean. Lange's handbook of chemistry (15th edn) (McGraw-Hill Inc., 1999) p. 4.1-4.84.

[12] С.А. Козюхин, К.Д. Цэндин, Х.Ф. Нгуен, В.В. Козик. Изв. вузов. Физика, 57 (7/2), 67 (2014).

[13] Y. Zhang, D. Ielminia. Appl. Phys. Lett., 90, 192102, (2007)

[14] Н. Мотт. Электроны в неупорядоченных структурах под ред. А.А. Бонч-Бруевича (М., Мир, 1967) с. 172.

[15] S. Kozyukhin, A. Sherchenkov, A. Babich, P. Lazarenko, H.P. Nguyen, O. Prikhodko. Canadian J. Phys., 92 (7/8), 684 (2014).

[16] M.H. Brodskiy. Topics Appl. Phys., 36 (1979).

[17] A. Van der Plass, R.H. Bube. J. Non-Cryst. Sol., 24, 377 (1976).
[18] P. Nagels, R. Callaerts, M. Denayer. Proc. 5th Int. Conf. Amorphous and Liquid Semiconductors, eds J. Stuke, W. Brenig (Taylor and Francis, London, 1973).

[19] G. Nicolescu, I. O’Connor, C. Piguet. Springer, 339 (2012).

[20] С.М. Зи. Физика полупроводниковых приборов (М., Энергия, 1973) с. 656 [пер. с англ. под ред. А.Ф. Трутко].

[21] М. Ламперт. Инжекционные токи в твердых телах (М., Мир, 1973) с. 416 [пер. с англ. под ред. С.М. Рывкина].

[22] A. Rose. Phys. Rev. 97 (6), 1538 (1955).

[23] L.F. Marsal, J. Pallare‘s, X. Correig. Appl. Phys., 79 (11), 8493 (1996).

[24] А.И. Исаев, С.И. Мехтиева, С.Н. Гарибова. ФТП, 45 (12), 1599 (2011).

[25] J. Weisfield. J. Appl. Phys., 54, 6401 (1983).

[26] Н.3. Джалилов, Г.М. Дамиров. ФТП, 43 (11), 1521 (2009).

[27] E. Prokhorov, J.J. Gervacio-Arciniega, G. Luna-Bárcenas, Y. Kovalenko, F.J. Espinoza-Beltrán, G. Trápaga. J. Appl. Phys., 113, 113705, (2013).

[28] J.M. Yahez-Limon, J. Gonzalez-Hernandez, J.J. Alvarado-Gil, I. Delgadillo, H. Vargas. Phys. Rev. B, 52 (23), 16321 (1995).

Редактор А.Н. Смирнов

\section{Electro-physical properties and transport mechanisms in phase change memory thin films on the basis of chalcogenide semiconductors on quasi-binary line $\mathrm{GeTe}-\mathrm{Sb}_{2} \mathrm{Te}_{3}$}

A.A. Sherchenkov ${ }^{1}$, S.A. Kozyukhin ${ }^{2}$, P.I. Lazarenko', A.V. Babich ${ }^{1}$, N.A. Bogoslovskiy ${ }^{3}$, I.V. Sagunova ${ }^{1}$, E.N. Redichev ${ }^{1}$

${ }^{1}$ National Research University of Electronic Technology, Zelenograd, 124498 Moscow, Zelenograd, Russia

${ }^{2}$ Kurnakov Institute of General and Inorganic Chemistry, Russian Academy of Sciences, 119991 Moscow, Russia

${ }^{3}$ loffe Institute, 194021 St. Petersburg, Russia

Abstract Temperature dependencies of resistivity and currentvoltage characteristics for phase change memory thin films on the basis of chalcogenide semiconductors on quasi-binary line $\mathrm{GeTe}-\mathrm{Sb}_{2} \mathrm{Te}_{3}: \mathrm{Ge}_{2} \mathrm{Sb}_{2} \mathrm{Te}_{5}, \mathrm{GeSb}_{2} \mathrm{Te}_{4}$ and $\mathrm{GeSb}_{4} \mathrm{Te}_{7}$ - were investigated. Influence of the composition variation along the quasi-binary line on the electro-physical characteristics and transport mechanisms of thin films was studied. Existence of three ranges with different current-voltage dependencies was established. Position and concentration of energy levels controlling charge transport were estimated. Obtained results show that electrophysical properties of thin films can be sufficiently varied by moving along the quasi-binary line $\mathrm{GeTe}-\mathrm{Sb}_{2} \mathrm{Te}_{3}$, which is important for the purposeful optimization of phase change memory technology. 